\title{
Modeling Microstructure Evolution in 718 Ingot to Billet Conversion
}

\author{
William Carden \\ Vista Engineering, Inc.; 2800 Milan Court, Suite115; Birmingham, AL 35211
}

\begin{abstract}
Alloy 718 billet production is a complex process intended to convert the as-cast ingot microstructure to a fine grain, uniform and equiaxed grain structure. For billets used in the production of turbine disks, meeting microstructural requirements consistently and efficiently is critical to billet producers. To this end, studies were conducted to construct a profile of recrystallization during intermediate stages of the conversion process. Two 6,000 pound VIMVAR ingots were processed normally through the first cogging pass and second upset operation. The partially transformed ingots were evaluated, modeled by finite element analysis, and sectioned for subsequent hot compression testing. A series of hot compression tests were used to replicate a range of thermo-mechanical conditions in the upsetting process. Quantitative microstructural information from the hot compression samples was used to create a collection of data for correlation of 718 microstructure evolution with the thermo-mechanical history of the material. Using this database, an empirical model was developed to predict microstructure evolution during 718 processing. A prototype software program is under development that would allow billet manufacturers to predict microstructures and optimize processing steps.
\end{abstract}

